\title{
Zur Verbesserung von Schlüsselkompetenzen in universitären Lehrveranstaltungen
}

\author{
Elmar Lange
}

Angenommen: 15. September 2011 / Online publiziert: 19. Januar 2012

(C) Institut für Arbeitsmarkt- und Berufsforschung 2012

Zusammenfassung Im Beitrag geht es um die empirisch zu beantwortende Frage, ob und unter welchen Bedingungen durch eigens eingerichtete universitäre Lehrveranstaltungen die von der OECD für wichtig gehaltenen Schlüsselkompetenzen „selbständiges Handeln“ und „Interagieren in sozial heterogenen Gruppen" sowie bestimmte Formen der Motivation und des Selbstbilds verbessert werden können. Im Ergebnis zeigt sich, dass Verbesserungen in den Schlüsselkompetenzen und in abgeschwächter Form auch in der Leistungsmotivation vor allem von der Qualität der Lehre der Dozenten abhängen, denen die Qualität des Lernens durch die Studierenden weitgehend folgt. Will man also die Schlüsselkompetenzen wie übrigens auch die fachlichen Kompetenzen der Studierenden verbessern, gilt es, die Qualität der Lehre durch geeignete Maßnahmen zu erhöhen.

\section{The improvement of key competencies in university courses}

\begin{abstract}
The article tries to answer the question empirically, whether and under which conditions the key competencies "acting autonomously" and "interacting in socially heterogeneous groups" as well as some forms of motivation and forms of the self concept can be improved by university courses, which are specially implemented for these reasons. It is a central result that the improvement of the key competencies and - to a smaller degree-of achievement motivation is depending on the quality of teaching, which is fol-
\end{abstract}

Zusätzliche Information ist in der online Version dieses Beitrags (doi:10.1007/s12651-012-0098-6) enthalten.

E. Lange ( $\varangle)$

Baumschulenweg 14, 48159 Münster, Deutschland

e-mail: Elmar.Lange@web.de lowed by the quality of learning of the students. Thus, to improve the key competencies as well as the professional competencies, it is necessary to improve the quality of teaching by effective means.

\section{Problem- und Fragestellungen}

Schlüsselkompetenzen für ein erfolgreiches Leben und eine gut funktionierende Gesellschaft stehen seit Ende der 90er Jahre im Zentrum der bildungstheoretischen und bildungspolitischen Vorstellungen der OECD (Rychen und Salganik 2001, 2003). Dort werden explizit drei Kernkompetenzbereiche definiert und vor dem Hintergrund allgemeiner gesellschaftlicher Anforderungen begründet: 1. selbstständiges Handeln, 2. Interagieren in sozial heterogenen Gruppen und 3. interaktive Nutzung von Instrumenten und Hilfsmitteln.

Jede dieser Schlüsselkompetenzen setzt die Mobilisierung von Wissen sowie von Einstellungen, Gefühlen, Werten und Motivation voraus, die zur Bewältigung komplexer situativer Anforderungen benötigt werden. Schlüsselkompetenzen gehen damit über Wissen und Fähigkeiten oder Fertigkeiten hinaus und kombinieren im Menschen liegende kognitive und evaluativ-motivationale Handlungsbedingungen mit externen situativen Anforderungen. Bei den Schlüsselkompetenzen geht es - vereinfacht gesagt - also nicht nur um „Wissen“, sondern um „Können“.

Mit dem Besitz von Schlüsselkompetenzen können nach den Vorstellungen der Vertreter dieses Ansatzes sowohl der individuelle Erfolg als auch die gesellschaftliche Wohlfahrt gefördert werden. Empirisch erfasst wurden ausgewählte Schlüsselkompetenzen international vergleichend bisher in den drei PISA-Studien 2000, 2003 und 2006. 
Mit dem Bologna-Abkommen zur Einführung gestufter Studiengänge in der EU von 1999 ist darüber hinaus für alle BA-Studiengänge vorgesehen, neben fachspezifischen Qualifikationen auch Schlüsselkompetenzen und Berufsorientierungen zu vermitteln.

Auch an der Universität Bielefeld werden in nahezu allen Fakultäten seit Einführung der BA-Studiengänge Veranstaltungen zur Förderung von Schlüsselkompetenzen und zur Berufsorientierung angeboten. In diesem Zusammenhang hat eine Lehrforschungsgruppe der Fakultät für Soziologie untersucht, inwieweit in ausgewählten einschlägigen Veranstaltungen im Wintersemester 2006/2007 Schlüsselkompetenzen vermittelt sowie Formen der Motivation und des Selbstbilds verändert worden sind.

In diesem Beitrag sollen die folgenden Fragen beantwortet werden:

1. Was verstehen wir unter den Schlüsselkompetenzen „Selbstständiges Handeln“ und „Interagieren in sozial heterogenen Gruppen“? Was verstehen wir unter Motivation und Selbstbild als eng mit Kompetenzen verbundenen Konzepten?

2. Welche Effekte haben die an der Universität eigens zum Zweck der Vermittlung von Schlüsselkompetenzen angebotenen Lehrveranstaltungen auf die Entwicklung der Schlüsselkompetenzen sowie auf die Entwicklung bestimmter Arten von Motivation und des Selbstbilds?

3. Welche hochschuldidaktischen Konsequenzen sind aus den Ergebnissen der Arbeit für die zukünftige Vermittlung von Schlüsselkompetenzen zu ziehen?

\section{Theoretische Überlegungen}

2.1 Was verstehen wir unter Schlüsselkompetenzen, Motivation und Selbstbild?

Bei der Auswahl und der Definition von Schlüsselkompetenzen lehnen wir uns eng an die Definition der OECD an (vgl. Rychen und Salganik 2001 und 2003): Schlüsselkompetenzen bezeichnen die Fähigkeiten von Menschen zur Bewältigung komplexer Anforderungen aus allen lebensweltlichen Bereichen. Hierzu gehören u.a. Anforderungen aus dem beruflichen, dem privaten sowie dem öffentlichen Bereich. Schlüsselkompetenzen verlangen den gleichzeitigen Einsatz kognitiver Fähigkeiten und evaluativer Orientierungen sowie deren ständiger Reflexion zur Bewältigung der situativen Anforderungen. Wir haben es hier also mit einer funktionalistischen Definition zu tun, wie sie auch der Kompetenzmessung in PISA zugrunde gelegt wurde.

Dem entsprechend unterscheiden wir hier die analytisch trennbaren, empirisch aber ineinander greifenden Schlüsselkompetenzbereiche:
1. Die Kompetenz zu selbstständigem Handeln, auch als autonome Handlungsfähigkeit bezeichnet, beinhaltet die folgenden Elemente:

- die Fähigkeit zur aktiven Orientierung in einer Handlungssituation, d.h. die Ermittlung der äußeren Bedingungen der Situation unter den Aspekten ihrer Anforderungen, Möglichkeiten und Restriktionen, die Ermittlung der inneren Bedingungen der Situation unter den Aspekten des eigenen Wissens, der eigenen Fähigkeiten und Fertigkeiten sowie der eigenen Bewertungen und Motivation sowie nicht zuletzt das reflektierende Inbeziehungsetzen der äußeren und inneren Bedingungen der Handlungssituation. Man kann diese Fähigkeit zur Entwicklung eines inneren Modells der Handlung in der Sprache der Rational Choice-Theorie auch als Fähigkeit zum Framing der Situation bezeichnen.

- die Fähigkeit zu ziel- bzw. zweckrationalem Handeln, d.h. die Zielsetzung, die Planung von Mitteln, die Umsetzung über Entscheiden und Handeln sowie die Bewertung anhand der Folgen. Darin eingeschlossen kann auch je nach im Framing-Prozess unterstelltem Modus ein wertrationales, traditionales oder affektuelles Handeln im Sinne von Max Weber sein.

- die Fähigkeit zu selbstgesteuertem Lernen mithilfe wirksamer Lernstrategien (z.B. Wiederholungs-, Elaborations- oder Kontrollstrategien).

2. Die Kompetenz zur Interaktion in sozial heterogenen Gruppen beinhaltet ebenfalls eine Reihe von Teilkompetenzen. Hierzu gehören zunächst einmal Fähigkeiten, die eigenen Interessen und Wünsche im sozialen Kontext auch zur Geltung zu bringen; man kann diese Fähigkeiten auch als egozentrischen Pol der Interaktionskompetenz bezeichnen. Im Einzelnen zählen hierzu:

- die Fähigkeit zur Verteidigung und Bestätigung der eigenen Interessen, Wünsche, allgemeinen und positionsgebundenen Rechte und Pflichten sowie Verantwortlichkeiten,

- die Fähigkeit zur Kritik und zur Offenheit gegenüber Kritik, sowie

- die Fähigkeit zu Konflikten und zur (friedlichen) Lösung von Konflikten im sozialen Kontext.

Auf der anderen Seite stehen Fähigkeiten, die man eher dem sog. allozentrischen Pol zurechnen kann; hierzu gehören:

- die Fähigkeit, Andersartigkeiten wahrzunehmen (Perspektivenübernahme),

- die Fähigkeit, sich in andere auch emotional hineinzuversetzen (Empathie),

- die Fähigkeit, gute und langfristig tragfähige Beziehungen zu anderen Menschen aufzubauen, sowie 
- die Fähigkeit, mit ihnen zu kooperieren.

3. Motivation zählt nicht direkt zu den Schlüsselkompetenzen. Aus handlungstheoretischen Überlegungen ist Motivation jedoch ein wesentlicher Bestandteil der Ressourcenausstattung einer Person in Situationen mit Anforderungscharakter. ${ }^{1}$ Motivation und Kompetenz sind analytisch trennbar, stehen empirisch aber in einem engen $\mathrm{Zu}$ sammenhang. ${ }^{2}$ Wir haben die folgenden Motivationsformen mit aufgenommen und empirisch untersucht:

- die intrinsische Motivation, die an den Inhalten von Lern- und Arbeitsprozessen und weniger an deren Folgen orientiert ist; letztere wird auch als extrinsische Motivation bezeichnet,

- die allgemeine Leistungsmotivation, in der in einer Aufgaben- bzw. Anforderungssituation die Hoffnung auf Erfolg die Furcht vor Misserfolg dominiert.

4. Das Selbstbild zählt zwar auch nicht direkt zu den Schlüsselkompetenzen. Es ist teils Bedingung, teils Folge ihres Einsatzes und kann deshalb als wesentliche Verstärkung der Kompetenzen begriffen werden. ${ }^{3}$ Im Einzelnen haben wir die folgenden Selbstbildkonzepte mit erhoben:

- die Selbstwirksamkeitswahrnehmung,

- die Selbstwertstärke,

- die Fähigkeit zur Selbstreflexion.

2.2 Von welchen Bedingungen hängt die Verbesserung der Schlüsselkompetenzen, der Motivation und des Selbstbilds in Lehrveranstaltungen der Universität ab?

Die Vermittlung von Schlüsselkompetenzen, Motivation und Selbstbild kann sich zunächst an einem allgemeinen pädagogisch-didaktischen Ansatz orientieren, der für all diese Ressourcen gilt. Hier haben wir ein Unterrichtskonzept herangezogen, das den Unterricht als einen Kommunikationsprozess begreift und das kurz wie folgt skizziert werden $\mathrm{kann}^{4}$ :

Der Unterricht hat zunächst einen Aufgabenbereich. Hier geht es um die Vermittlung und den Erwerb von

\footnotetext{
${ }^{1}$ Vgl. für die psychologischen Motivationstheorien: Heckhausen (1963, 1977, 1989), Deci (1975), Deci und Ryan (2000), Schiefele und Köller (2006); für die soziologische Handlungstheorie: z.B. Esser (1999).

${ }^{2}$ Dabei ist unseres Wissens weder in der psychologischen noch in der soziologischen Handlungstheorie geklärt, ob es sich um reine Kovariations- oder um Kausalitätsverhältnisse handelt.

${ }^{3}$ Eine systematische Einführung in die Selbstkonzeptforschung bietet Mummendey (2006).

${ }^{4}$ Vgl. Lange (1992, 2005). Ganz ähnliche Überlegungen finden sich bereits in Fittkau (1979) sowie in Hennig (2006). Eine ausführliche Darstellung erfolgt im 3. Abschnitt.
}

Kompetenzen. Hier stehen Lehrende und Studierende einander als Experten bzw. als Lernende gegenüber. Der Kommunikationsprozess stellt einen Lehr-Lern-Prozess dar, der auf Seiten der Lernenden als Informationsaufnahme-, Informationsverarbeitungs- und -anwendungsprozess betrachtet werden kann. Im Vordergrund stehen Tüchtigkeitsaspekte sowohl auf Seiten der Lehrenden wie der Lernenden. Was die Methodik und Didaktik angeht, hat die Unterrichtsforschung angesichts der Fülle der Möglichkeiten hier das für manche beruhigende, für manche enttäuschende Ergebnis erbracht, dass es die einzig wahre Methodik und Didaktik nicht gibt, sondern dass allenfalls ein bestimmter Wechsel die Ergebnisse zu optimieren vermag (Fend 1980, S. 307; Lange 2005, S. 174ff.).

Der Unterricht hat weiterhin einen sozial-emotionalen Bereich. Dort geht es um Persönlichkeitsbildung, d.h. auch um die Vermittlung und Prägung von Werthaltungen und Einstellungen. Hier stehen Lehrende und Studierende einander als Vorbilder bzw. als kritische Partner gegenüber. Der Vermittlungsprozess ist hier ein Kommunikationsprozess, in dem zumindest schwach positive sozial-emotionale Beziehungen auf einer Vertrauensbasis gepflegt werden müssen, damit auf der anderen Ebene die Vermittlung und Übernahme fachlicher und überfachlicher Kompetenzen auch gelingt. Im Vordergrund stehen hier sog. Beliebtheitsmerkmale der Lehrenden und Studierenden.

Dass darüber hinaus situative, also organisatorische, unterrichtliche, personale, finanzielle und materiale Bedingungen für eine ,erfolgreiche“ Vermittlung von Kompetenzen, Motivation und Selbstbildern berücksichtigt werden sollten, zeigen die Ergebnisse der Schul- und Hochschulforschung. In dieser Studie berücksichtigen wir nur die Art der konkret untersuchten Lehrveranstaltungen der verschiedenen Fakultäten.

Was die internen Bedingungen ,erfolgreichen Unterrichts" angeht, die sich hier in Kompetenz-, Motivationsund Selbstbildverbesserungen niederschlagen, betrachten wir zum einen das Verhalten der Lehrenden, wie es aus der Perspektive der Studierenden eingeschätzt wird. Dabei wird der Lehrende zum einen als Fachmann, als Experte betrachtet, der bestimmte Tüchtigkeitsmerkmale aufweisen muss, um im Unterricht als einem Informationsprozess im sog. Aufgabenbereich fachliche Verbesserungen, Kenntnisverbesserungen zu erreichen, oder auch Lernprozesse in Gang zu setzen; hierzu gehört auch die Förderung von Schlüsselkompetenzen.

$\mathrm{Zu}$ diesen Tüchtigkeitsmerkmalen zählen wir die folgenden Eigenschaften und Verhaltensweisen des Lehrenden:

- die fachliche Kompetenz, die sich aus seinem Wissen, seiner rhetorischen Kompetenz, einschließlich der Klarheit und Verständlichkeit in seiner Darstellung zusammensetzt,

- die didaktische Kompetenz, die sich aus guten Erklärungen und Zusammenfassungen, gegenstandsadäquatem 
Abb. 1 Unterricht als Kommunikationsprozess

\begin{tabular}{|c|c|c|c|c|}
\hline $\begin{array}{l}\text { Situative } \\
\text { Bedingungen }\end{array}$ & & Unterricht & \multicolumn{2}{|c|}{$\begin{array}{l}\text { individuelle } \\
\text { Folgen }\end{array}$} \\
\hline & $\begin{array}{l}\text { Tüchtigkeits- } \\
\text { verhalten } \\
\text { des Lehrenden }\end{array}$ & & \multicolumn{2}{|c|}{$\begin{array}{l}\text { Tüchtigkeits- } \\
\text { verhalten } \\
\text { des Studierenden }\end{array}$} \\
\hline & & $\begin{array}{l}\text { Aufgabenbereich } \\
\text { fachliche Entwicklung }\end{array}$ & & \\
\hline materielle & als Experte & Informationsprozess & als Lernender & $\begin{array}{l}\text { „Leistung“" } \\
\text { Kompetenz }\end{array}$ \\
\hline \multirow[t]{3}{*}{ Bedingungen } & als „Mensch“ & sozialer Prozess & als „Mensch“ & $\begin{array}{l}\text { Werte } \\
\text { Einstellung }\end{array}$ \\
\hline & & $\begin{array}{l}\text { Sozial-emot. Bereich } \\
\text { Persönlichkeits- } \\
\text { Bildung }\end{array}$ & & \\
\hline & $\begin{array}{l}\text { soziales } \\
\text { Verhalten } \\
\text { des Lehrenden }\end{array}$ & & \multicolumn{2}{|c|}{$\begin{array}{l}\text { Verhalten } \\
\text { des Studierenden }\end{array}$} \\
\hline
\end{tabular}

Tempo und auf das Vorwissen bezogenem Schwierigkeitsgrad zusammensetzt, und

- die Strukturiertheit der Veranstaltung, die sich in einer klaren Gliederung und Ordnung des Stoffs niederschlägt.

Zum anderen muss der Lehrende bestimmte Beliebtheitsmerkmale aufweisen, um im Unterricht im sozial-emotionalen Bereich zur Persönlichkeitsentwicklung der Studierenden beizutragen. Hierzu gehören dann auch die Förderung von Lernmotivation, Leistungsmotivation und die Verbesserung der Selbstwahrnehmung.

$\mathrm{Zu}$ diesen Beliebtheitsmerkmalen zählen wir die folgenden Verhaltensweisen:

- der Respekt, mit dem er den Studierenden begegnet; hierzu zählen ein freundlicher Umgang mit ihnen und sie ernst zu nehmen,

- die Motivierung der Studierenden; hierzu zählen die Erzeugung von Interesse am Gegenstand, die lebendige Gestaltung des Unterrichts und die Schaffung einer angenehmen Atmosphäre bzw. eines positiven Unterrichtsklimas,

- die ausreichende Betreuung der Studierenden vor und nach den Veranstaltungen, sowie

- der Grad der Förderung von Kooperation und Gruppenarbeit.

$\mathrm{Zu}$ den internen Bedingungen ,erfolgreichen Unterrichts“ gehören aber auch komplementäre Verhaltensweisen der Studierenden. Sie werden zum einen als Lernende begriffen, die im Unterricht bestimmte Tüchtigkeitsverhaltensmuster zeigen müssen, um erfolgreich zu sein.

$\mathrm{Zu}$ diesen studentischen Tüchtigkeitsmerkmalen zählen wir die folgenden Verhaltensweisen:

- der Umfang und die Qualität der aktiven schriftlichen Verarbeitung der Veranstaltung, einschließlich des Um- fangs und der Qualität der Vor- und Nachbereitung der Veranstaltung,

- der Umfang, in dem sie sich aktiv in Veranstaltungen durch Fragen und Diskussionsbeiträge einbringen,

- der Umfang, in dem sie sich mit Freunden und Bekannten über die Veranstaltung unterhalten, d.h. in welchem Umfang ein Transfer in außeruniversitäre soziale Gruppen erfolgt, sowie

- die Zahl der eigenen Fehlstunden.

Die Studierende werden aber auch mit ihren sozial-emotionalen Verhaltensweisen begriffen, von denen wir hier nur

- das Ausmaß der konstruktiven Mitarbeit der Studierenden insgesamt und

- das Ausmaß des Desinteresses bzw. das Ausmaß der Unterrichtsstörungen der Studierenden, wie Unaufmerksamkeit, Reden, Herausgehen

erfasst haben.

Der Unterricht wird damit als ein zwischen Lehrenden und Studierenden ablaufender Kommunikationsprozess betrachtet, in dem Lehrende und Studierende sowohl unter fachlichen Aspekten im Rahmen eines Lehr-Lernprozesses als auch unter sozial-emotionalen Aspekten im Rahmen eines grundlegenden sozial-emotionalen Prozesses miteinander interagieren. Nur wenn die für beide Prozessbereiche erforderlichen komplementären Verhaltensmuster von beiden Seiten an den Tag gelegt werden, erscheint ein „erfolgreicher" Unterricht möglich.

Vereinfacht lassen sich diese Überlegungen in den folgenden Abbildungen darstellen, wobei Abb. 1 den Unterricht als Kommunikationsprozess begreift und Abb. 2 die 
Abb. 2 Kausalmodell zur Erklärung „erfolgreichen“ Unterrichts

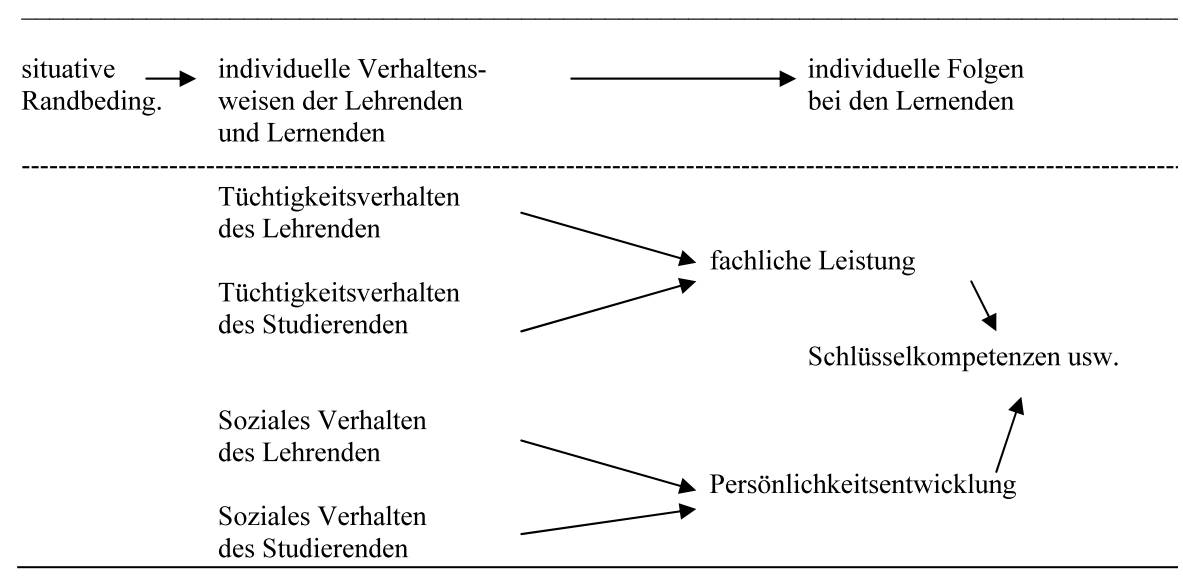

\begin{tabular}{|c|c|c|c|}
\hline & $\mathrm{T} 1$ & $\mathrm{~T} 2$ & T3 \\
\hline \multirow{4}{*}{$\begin{array}{l}\text { Kontrollgruppe } \\
\text { Kontimentalgruppe }\end{array}$} & M1 & X (Lehre) & M2 \\
\hline & & & M3 \\
\hline & Beginn WS & lfd. WS & Ende WS \\
\hline & $2006 / 07$ & $2006 / 07$ & $2006 / 07$ \\
\hline
\end{tabular}

M1 - M3 = Messung/Erhebung
Abb. 3 Methodisches Design zur experimentellen Impactevaluation
Umsetzung der kommunikationstheoretischen Erörterungen in ein empirisch testbares Kausalmodell darstellt. ${ }^{5}$

An dieser Stelle bleibt darauf hinzuweisen, dass eine gültige Untersuchung der Bedingungen von Schlüsselkompetenzen als einer speziellen Form von Kompetenzen nicht nur empirisch möglich ist, sondern auch unter Anwendung von Regeln der Logik aus einer allgemeineren anerkannten hochschuldidaktischen Theorie der Vermittlung von Kompetenzen durchgeführt werden kann.

\section{Methodischer Ansatz}

\subsection{Welches Forschungsdesign wurde gewählt?}

Für eine empirische Beantwortung der Forschungsfragen bzw. für einen empirischen Test des hier vorliegenden theoretischen Modells wird ein experimentelles Forschungsdesign benötigt (vgl. Abb. 3).

Benötigt wird eine Längsschnitterhebung der Studierenden vor und nach dem Besuch von Lehrveranstaltungen zur

\footnotetext{
${ }^{5}$ An dieser Stelle sei nur kurz darauf hingewiesen, dass sich die vorgestellten kommunikationstheoretischen Überlegungen auch sehr gut in ein allgemeines handlungstheoretisches Modell integrieren lassen, wie es in der aufgeklärten Version der Rational Choice-Theorie vorliegt [für die hier vorliegende Studie: Lange (2009), S. 27ff., allgemein: Esser (1999), Coleman (1995)].
}

Vermittlung von Schlüsselkompetenzen (Experimentalgruppe) sowie eine Erhebung von Studierenden einer Lehrveranstaltung nach der Veranstaltung, in der aber nicht bewusst Schlüsselkompetenzen vermittelt werden (Kontrollgruppe).

Darüber hinaus werden für die Studierenden in der Experimentalgruppe die ,erfolgsrelevanten“ situativen Merkmale der Lehrveranstaltung, das Lehrenden- und das Lernendenverhalten erhoben. Mit diesem experimentellen Ansatz lassen sich Veränderungen in den Schlüsselkompetenzen der Studierenden auf die Teilnahme an dieser Lehrveranstaltung zurückführen, wenn sich zum einen die Vorherund Nachhermessungen (M1 und M2) der Schlüsselkompetenzen signifikant unterscheiden und sich zum anderen keine Unterschiede zwischen den Schlüsselkompetenzen der Experimentalgruppe in der Vorhermessung (M1) und der Kontrollgruppe (M3) ergeben. Darüber hinaus können unter Rückgriff auf die situativen Bedingungen die Veränderungen in den Kompetenzen genauer spezifiziert werden. Nicht zuletzt lassen sich - nach Bildung von Differenzvariablen aus Vor- und Nacherhebungen - diejenigen identifizieren, die entweder keine oder aber mehr oder weniger große Lernfortschritte bei den Schlüsselkompetenzen erreichen. Auch diese Lernfortschritte lassen sich dann aus den Randbedingungen und aus den situativen Bedingungen erklären.

\subsection{Wer sind die Untersuchungseinheiten?}

Als Untersuchungseinheiten der Experimentalgruppe dienten alle Studierenden aus den Lehrveranstaltungen der Fa- 
Tab. 1 Studierende in den Experimental- und Kontrollgruppen

\begin{tabular}{lllll}
\hline Gruppen-Code & Tag/Zeit & Vorher & Nachher & Vorher und Nachher \\
\hline 111 & mo 08-10 & 40 & 14 & 25 \\
121 & mo 10-12 & 42 & 15 & 15 \\
131 & di 14-16 & 40 & 31 & 21 \\
141 & do 08-10 & 31 & 23 & 7 \\
151 & do $10-12$ & 34 & 29 & 12 \\
211 & mo $12-14$ & 56 & 38 & 28 \\
311 & fr $10-12$ & 44 & 33 & 25 \\
321 & mi $10-12$ & 35 & 33 & 24 \\
331 & mi $14-16$ & 16 & 17 & 11 \\
ExGr gesamt & & 338 & 233 & 168 \\
KoGr & di $10-12$ & 46 & & \\
\hline
\end{tabular}

ExGr voher + KoGr: $n=384$

kultät für Soziologie, der Fakultät für Geschichtswissenschaften und der Fakultät für Literaturwissenschaften und Linguistik im WS 2006/2007, die von dem zentralen Servicebereich bei der Vermittlung von Schlüsselkompetenzen unterstützt wurden. Hierzu zählten sechs mit Tutoren durchgeführte Einführungsveranstaltungen aus dem Bereich der Politikwissenschaft, eine Veranstaltung aus dem Bereich der Anglistik und drei Veranstaltungen aus dem Bereich der Geschichtswissenschaften. Als Kontrollgruppe wurde eine Einführungsveranstaltung aus der Fakultät für Soziologie gewählt, an der Studierende der Politikwissenschaft sowie der Sozialwissenschaften teilnahmen. Damit ergibt sich nach Abschluss der Erhebungen die folgende Verteilung der Studierenden (vgl. Tab. 1).

Insgesamt haben damit 338 Studierende an der Erst- und 233 Studierende an der Zweiterhebung in der Experimentalgruppe teilgenommen, das entspricht einem Teilnehmerschwund von etwa einem Drittel aller Studierenden. An beiden Erhebungen haben insgesamt nur 168 Studierende, also nur die Hälfte derjenigen teilgenommen, die auch an der Ersterhebung beteiligt waren.

\subsection{Wie kann man Schlüsselkompetenzen und ihre} Determinanten gültig erheben?

Da wir uns entschlossen haben, uns eng an die Schlüsselkompetenzkonzeption der OECD anzuschließen, wie sie ansatzweise auch schon in den PISA-Studien umgesetzt wurde, müssen wir bei der Instrumentenerstellung auch nicht in jedem Fall von vorne beginnen. Die Schlüsselkompetenzen wurden in dieser Studie zum größten Teil über Skalen, zum geringeren Teil über Einzelitems und über Indexbildungen erhoben. Einen großen Teil der Skalen konnten wir dabei der „Pädagogischen Jugend- und Rekrutenbefragung ch- $\mathrm{x}$ “ entnehmen, einer Studie, die im Forschungsbereich Schulqualität und Schulentwicklung des Pädagogischen Institu- tes der Universität Zürich durchgeführt wurde bzw. weiterhin durchgeführt wird (Buschor und Forrer 2005). Im Rahmen dieser Studie ging es u.a. explizit um die Entwicklung und Validierung von Instrumenten zur Erfassung überfachlicher Kompetenzen, die den Schlüsselkompetenzen der OECD sehr ähnlich sind (vgl. Grob und Maag Merki 2001, 2003). Weiterhin wurde die Vermittlung von Schlüsselkompetenzen durch die Studierenden selbst eingeschätzt.

Die sich aus dem theoretischen Modell ergebenden Determinanten wurden teils über Fragen, teils über Skalen erhoben, die sich in früheren eigenen Untersuchungen (u.a. Lange 1992, 2004) bewährt hatten. ${ }^{6}$

Die Erhebung erfolgte mittels weitgehend standardisierter Fragebögen während der Lehrveranstaltungen in der Universität. Die durchschnittliche Bearbeitungszeit lag zwischen 45 und 60 Minuten. Alle Fragebögen wurden anschließend kodiert und die Daten in SPSS-Files übertragen, die der weiteren Analyse zugrunde liegen.

\subsection{Wie wurden die Daten ausgewertet?}

Alle übernommenen und selbst gebildeten Skalenitems zu den Schlüsselkompetenzen, zur Motivation und Selbstwahrnehmung wurden über Faktorenanalysen und Reliabilitätsanalysen auf ihre Skalierbarkeit hin überprüft. Die Zusammenhänge zwischen den latenten und manifesten Variablen der Kompetenzen, Motivationsarten und Formen der Selbstwahrnehmung wurden darüber hinaus über konfirmatorische Faktorenanalysen (mit Hilfe von Lisrel) untersucht. Andere Items, insbesondere zu den unabhängigen und intervenierenden Variablen, wurden entweder zu Indizes verarbeitet oder als Einzelitems übernommen. Nach Überprüfung der einfachen Häufigkeitsverteilungen aller Skalen, Indizes und Einzelitems wurden, ggf. nach Rekodierungen, den Hypothesen des theoretischen Modells entsprechend, zweidimensionale Häufigkeitsverteilungen und die zugehörigen $\mathrm{Zu}$ sammenhangsmaße errechnet. Dargestellt werden der Einfachheit halber vor allem die Korrelationskoeffizienten. ${ }^{7}$ Zur Überprüfung der Veränderungen in den Schlüsselkompetenzen wurde der T-Test für abhängige bzw. für unabhängige Stichproben gewählt. Darüber hinaus wurden Varianzanalysen gerechnet. $^{8}$

\footnotetext{
${ }^{6}$ Sämtliche Operationalisierungen finden sich im Electronic Supplementary Material, Online Resource 1.

${ }^{7}$ Wenn wir hier auf Pearson's $r$ als Zusammenhangsmaß abstellen, statt die häufig angemesseneren Maßzahlen für ordinal-skalierte Variablen zu verwenden, geschieht das aus Gründen der besseren Vergleichbarkeit der Ergebnisse. Durchgeführte Vergleiche etwa zwischen $r$ und Kendall's Tau b oder c zeigen durchweg nur geringe Unterschiede.

${ }^{8}$ Die entsprechenden Tabellen finden sich im Supplementary Material, Online Resource 2.
} 


\section{Ergebnisse}

Im Folgenden sollen ausgewählte Ergebnisse zu den folgenden vier Fragen präsentiert werden:

1. Werden in den von der zentralen Serviceeinrichtung der Universität unterstützten Lehrveranstaltungen die Schlüsselkompetenzen sowie die Motivation und das Selbstbild während des Untersuchungszeitraums signifikant verbessert, wie es hochschulpolitisch gewünscht ist?

2. Gibt es Zusammenhänge zwischen den Veränderungen in den Schlüsselkompetenzen, der Motivation und dem Selbstbild einerseits und den Lehr- bzw. Lernverhaltensweisen der Lehrenden und der Studierenden? Untersuchungseinheiten sind hier Personen/Studierende.

3. Unterscheiden sich die einzelnen Lehrveranstaltungen hinsichtlich der Veränderungen der Kompetenzen, der Motivation und des Selbstbilds, also hinsichtlich möglicher Verbesserungen bzw. Verschlechterungen? Untersuchungseinheiten sind hier Veranstaltungen.

4. Gibt es Zusammenhänge zwischen den Veränderungen der Kompetenzen, der Motivation und des Selbstbilds (Verbesserungen, Verschlechterungen) und der Qualität des Lehrens bzw. des Lernens in den einzelnen Veranstaltungen? Untersuchungseinheiten sind auch hier Veranstaltungen.

5. Zum Schluss soll noch kurz die Frage beantwortet werden: Kann von der Zufriedenheit der Studierenden mit den Veranstaltungen bzw. den Veranstaltern auf die Qualität der Lehre geschlossen werden?

\subsection{Werden durch die Lehrveranstaltungen die} Schlüsselkompetenzen sowie die Motivation und das Selbstbild während des Untersuchungszeitraums insgesamt verbessert?

Hier geht es um einen Vergleich der Ergebnisse der Messungen in der Experimentalgruppe zu Beginn der Veranstaltung (M1) mit den Messungen am Ende der Veranstaltungen (M2) sowie den Vergleich von M1 mit M3 (Kontrollgruppe); das statistische Verfahren sind T-Tests für gepaarte Stichproben (M1 vs. M2) und T-Tests für unabhängige Stichproben (M1 vs. M3). Die Ergebnisse finden sich im supplementary material, online resource 2, Tab. A1-A8.

Beide Testverfahren zeigen eindeutig:

Über alle Veranstaltungen gesehen gibt es keine signifikanten Verbesserungen

- bei der Kompetenz zum selbstständigen Handeln,

- bei der Interaktionskompetenz,

- bei der Motivation und

- beim Selbstbild.

Dieses Ergebnis war aus soziologischer Perspektive aus zumindest drei Gründen zu erwarten:
- Die kurzen einsemestrigen Lehrveranstaltungen können im Vergleich zu den langjährigen Sozialisationsprozessen, die die Studierenden im Elternhaus, in der Schule und in den Freundesgruppen erfahren haben, allenfalls einen minimalen zusätzlichen Effekt besitzen. Das wird auch in der Einschätzung der Studierenden hinsichtlich des Stellenwertes der verschiedenen Sozialisationsinstanzen zur Förderung der Schlüsselkompetenzen deutlich: Der Hochschule wird diesbezüglich die geringste Bedeutung zugemessen.

- Die Lehrenden der untersuchten Veranstaltungen wurden nur in wenigen Stunden in der Vermittlung der Kompetenzen durch Mitglieder der zentralen Serviceeinrichtung ausgebildet. Ob eine derartige Ausbildung sich auch bei der Durchführung der Kurse nachhaltig niederschlägt, muss ebenfalls bezweifelt werden.

- Die Ausbildung der Lehrenden durch die Mitglieder der Serviceeinrichtung richtete sich auch weniger auf die Förderung der hier untersuchten Schlüsselkompetenzen, Motivation und Selbstwahrnehmung als auf die Förderung der Studierenden im Umgang mit wissenschaftlichen Arbeitstechniken.

Damit ist aber noch nicht gesagt, dass sich nicht in den einzelnen Veranstaltungen Veränderungen (Verbesserungen, Verschlechterungen) ergeben haben, die sich insgesamt neutralisieren; wir kommen darauf bei der Einzelanalyse der Veranstaltungen zurück.

4.2 Gibt es Zusammenhänge zwischen den Veränderungen in den Schlüsselkompetenzen, der Motivation und Selbstwahrnehmung einerseits und den Lehr- bzw. Lernverhaltensweisen der Lehrenden und der Studierenden?

Die Veränderungen in den Kompetenzen, der Motivation und der Selbstwahrnehmung wurden über Differenzvariablen gemessen, in denen die Ersterhebung von der Zweiterhebung subtrahiert wurde.

\subsubsection{Die Kompetenz zu selbstständigem Handeln}

Wie die Veränderung der Kompetenz zu selbstständigem Handeln von den Verhaltensweisen der Lehrenden abhängt, zeigt Tab. 2 in Form von Korrelationskoeffizienten (vgl. Tab. 2).

Die Kompetenz der Studierenden zum selbstständigen Handeln steigt umso mehr,

- je strukturierter die Veranstaltung abläuft, je klarer also ihre Ordnung, in der ein roter Faden erkennbar ist, in der klare Fragen gestellt und systematisch beantwortet bzw. Probleme formuliert und systematisch gelöst werden, 
Tab. 2 Zusammenhänge zwischen den Veränderungen der Kompetenzen zum selbstständigen Handeln und den Verhaltensweisen der Lehrenden in den Kursen

\begin{tabular}{llccc}
\hline & $\begin{array}{l}\text { Veränd. selbst. } \\
\text { Handeln } r\end{array}$ & $\begin{array}{l}\text { Veränd. aktive } \\
\text { Orientierung } r\end{array}$ & $\begin{array}{l}\text { Veränd. zweckrat. } \\
\text { Handeln } r\end{array}$ & $\begin{array}{l}\text { Veränd. selbstreg. } \\
\text { Lernen } r\end{array}$ \\
\hline Kompetenz &, 201 &, 049 &, 187 &, 140 \\
Didaktik &, 076 &, 041 &, 030 &, 102 \\
Strukturierung &, 253 &, 087 &, 139 &, 281 \\
Respektierung &, 161 &, 076 &, 168 &, 062 \\
Motivierung &, 191 &, 175 &, 155 &, 080 \\
Betreuung &, 074 &, 013 &, 042 &, 133 \\
Gruppenarbeit &, 150 &,- 005 &, 149 & 163 \\
$n$ & 161 & 165 & 164 & 164 \\
\hline
\end{tabular}

Korrelationskoeffizienten $r>.15=$ sign. auf 95\%-Niveau, Koeff. $>.20=$ sign. auf 99\%-Niveau

Tab. 3 Zusammenhänge zwischen den Veränderungen der Kompetenzen zum selbstständigen Handeln und den Verhaltensweisen der Studierenden in den Veranstaltungen

\begin{tabular}{lcccc}
\hline & $\begin{array}{l}\text { Veränd. selbst. } \\
\text { Handeln } r\end{array}$ & $\begin{array}{l}\text { Veränd. aktive } \\
\text { Orientierung } r\end{array}$ & $\begin{array}{l}\text { Veränd. zweckrat. } \\
\text { Handeln } r\end{array}$ & $\begin{array}{l}\text { Veränd. selbstreg. } \\
\text { Lernen } r\end{array}$ \\
\hline Vor-Nachbereitung &, 101 &, 053 &, 097 &, 049 \\
aktive Mitarbeit &, 080 &, 043 &, 088 &, 027 \\
Transfer &, 200 &, 151 &, 113 &, 175 \\
Gruppenmitarbeit &,- 022 &,- 012 &, 014 &,- 044 \\
Gruppendesinteresse &,- 089 &,- 027 &,- 150 &,- 017 \\
Semesterfehlstunden &, 016 &,- 081 &, 024 &, 036 \\
$n$ & 163 & 167 & 166 & 166 \\
\hline
\end{tabular}

Korrelationskoeffizienten $r>.10=$ sign. auf $95 \%$-Niveau, Koeff. $>.13=$ sign. auf 99\%-Niveau

- je höher die Kompetenz des Lehrenden in fachlicher und rhetorischer Hinsicht eingeschätzt wird; dazu gehört auch eine klare und verständliche Darstellung des Stoffs,

- je stärker der Lehrende die Studierenden zu motivieren und ihr Interesse zu wecken und zu erhalten versteht,

- je größer der Respekt, den der Lehrende seinen Studierenden entgegenbringt und

- je häufiger er auch Gruppenarbeit zulässt.

- Eine eher untergeordnete Rolle scheinen nach diesen Ergebnissen die didaktische Qualität und der Umfang der Betreuung zu spielen.

Fragen wir jetzt, wie die Veränderung der Kompetenz zu selbständigem Handeln von den Studierverhaltensweisen abhängt (vgl. Tab. 3).

Entgegen unseren Erwartungen besitzen die selbst eingeschätzten studentischen Verhaltensweisen, also ihr Tüchtigkeitsverhalten und ihr Sozialverhalten, im Vergleich zum Lehrendenverhalten eine deutlich geringere Bedeutung für die Kompetenzveränderungen. Festzuhalten bleibt:

- Die Kompetenz zum selbstständigen Handeln wird insbesondere dann gestärkt, wenn sich die Studierenden über die Veranstaltungen hinaus mit ihren Mitstudierenden oder Freunden und Bekannten über den Stoff unterhalten und ihn in diesen Kreisen intensiv diskutieren. Dieser Transfer erweist sich für die Kompetenz zum selbstständigen Handeln insgesamt und für alle Teilkompetenzen als gleichermaßen relevant.

- Die Vor- und Nachbereitung der Veranstaltungen sowie die aktive Mitarbeit erhöhen zwar die Kompetenz zu selbstständigem Handeln; die Zusammenhänge sind aber nicht signifikant.

- Desinteresse der Mitstudierenden an den Veranstaltungen, das sich in Störungen, eigenen Gesprächen usw. ausdrückt, ist erwartungsgemäß auch der Kompetenzentwicklung nicht förderlich; allerdings sind die Zusammenhänge insgesamt nur schwach.

\subsubsection{Veränderungen in der Interaktionskompetenz}

Erwartungsgemäß sind die Zusammenhänge zwischen den Verbesserungen der Interaktionskompetenzen der Studierenden und der Qualität der Lehre in den Kursen eher 
schwach ausgeprägt und darüber hinaus hinsichtlich der Teilkompetenzen inkonsistent. Im Einzelnen:

- Die Interaktionskompetenz der Studierenden wird durch die Qualität der Lehre insgesamt nicht signifikant erhöht, weil sich positive und negative Zusammenhänge bei den Teilkompetenzen neutralisieren.

- So steigt die egozentrische Kompetenz, also die Kompetenz, in Interaktionen die eigenen Interessen und Rechte einzubringen, Kritik zu äußern und zu akzeptieren, deutlich mit den sozialen Verhaltensweisen der Lehrenden, weniger mit ihren auf Tüchtigkeit abstellenden Verhaltensweisen. Die Verbesserung der egozentrischen Kompetenz der Studierenden ist umso höher, je stärker die Dozenten sie zu motivieren vermögen $(r=.15 \mathrm{~s})$, je mehr Respekt sie ihnen entgegenbringen $(r=.19 \mathrm{~s})$ und je intensiver sie sie betreuen $(r=.16 \mathrm{~s})$.

- Für die Verbesserung der allozentrischen Kompetenzen gilt tendenziell das Gegenteil, wenngleich die Zusammenhänge hier nicht signifikant sind.

Die Ergebnisse zu den Effekten der eigenen Verhaltensweisen der Studierenden sind auf den ersten Blick ernüchternd. Bei der Interaktionskompetenz werden nur Teilkompetenzen im allozentrischen Bereich verbessert:

- Die Kooperationsfähigkeit steigt, je konstruktiver die Mitarbeit der Kommilitonen und je regelmäßiger man an den Veranstaltungen teilgenommen hat $(r=.19 \mathrm{~s} \mathrm{bzw}$. $r=.20 \mathrm{ss})$.

- Die Empathie, also die Fähigkeit, sich in andere hineinzuversetzen, steigt ebenfalls mit dem Umfang der konstruktiven Mitarbeit der Kommilitonen $(r=.19 \mathrm{~s})$ und

- die Fähigkeit zur Perspektivenübernahme steigt in dem Maße, in dem man sich auch außerhalb der Veranstaltung mit seinen Kommilitonen austauscht $(r=.15 \mathrm{~s})$.

Damit ergibt sich ein einigermaßen konsistentes Bild der hier untersuchten Teilkompetenzen: Die Kooperationsfähigkeit, die Fähigkeiten zum Perspektivenwechsel und zur Empathie stehen in engem Zusammenhang mit der Einbindung der Studierenden in den Kreis der Kommilitonen im und außerhalb des Unterrichts. Je intensiver diese Einbindung, je produktiver und konstruktiver die Gruppe, desto stärker wachsen auch die Interaktionskompetenzen.

\subsubsection{Veränderungen in der Motivation und der Selbstwahrnehmung und ihre unterrichtliche Bedingungen}

Im Motivationsbereich wird nur die Leistungsmotivation durch die Lehr- und Lernverhaltensweisen verbessert:

- Die Leistungsmotivation der Studierenden steigt umso mehr, je mehr die Lehrenden die Studierenden zu motivieren und ihr Interesse zu wecken vermögen und je an- genehmer und je lebendiger sie das Unterrichtsklima gestalten $(r=.13)$.

- Die Leistungsmotivation steigt direkt mit dem Ausmaß der Mitarbeit der Studierenden in den Veranstaltungen, insbesondere mit dem Umfang, in dem sie während des Unterrichts mitschreiben und den Unterricht vor- und nachbereiten, und mit dem Umfang, in dem auch die anderen Studierenden Interesse zeigen und mitarbeiten (jeweils $r=.15 \mathrm{~s}$ ).

Was die Selbstwahrnehmung angeht, zeigen sich die folgenden Ergebnisse:

- Die Veränderungen der Wahrnehmung der Selbstwirksamkeit sind weder durch das Lehrverhalten der Lehrenden noch durch das Lernverhalten der Studierenden zu erklären.

- Das Selbstwertgefühl der Studierenden steigt mit dem Respekt, den die Lehrenden den Studierenden entgegenbringen $(r=.16 \mathrm{~s})$, und sinkt mit ihren eigenen Fehlzeiten $(r=.21 \mathrm{ss})$.

- Die Fähigkeit zur Selbstreflexion steigt umso mehr, je mehr die Lehrenden Gruppenarbeit unterstützen $(r=$ $.17 \mathrm{~s})$.

4.3 Unterscheiden sich die einzelnen Lehrveranstaltungen hinsichtlich der Veränderungen der Kompetenzen, Motivation und Selbstwahrnehmung, also hinsichtlich möglicher Verbesserungen bzw. Verschlechterungen?

Um diese Frage zu beantworten, haben wir Varianzanalysen durchgeführt, in die die Kursvariable als Faktor eingeht. Die Ergebnisse finden sich im supplementary material, resource 2 Tab. A9-A12.

- Hinsichtlich der Kompetenz zum selbstständigen Handeln zeigen sicherhebliche Differenzen zwischen den einzelnen Lehrveranstaltungen: So finden wir überdurchschnittliche Kompetenzverbesserungen in den Veranstaltungen 131 und 311 und überdurchschnittliche Kompetenzverschlechterungen in den Veranstaltungen 141 und 321, die übrigen Veranstaltungen liegen dazwischen. Die Varianzanalyse belegt darüber hinaus, dass die Unterschiede zwischen den Veranstaltungen hochsignifikant sind.

- Hinsichtlich der Interaktionskompetenz zeigen sich deutliche Verbesserungen in den Veranstaltungen 121 und 131, während in den Veranstaltungen 111, 141 und 151 überdurchschnittliche Verschlechterungen auftreten. Auch hier belegt die Varianzanalyse, dass die Unterschiede zwischen den Veranstaltungen bei der Interaktionskompetenz insgesamt sowie bei der allozentrischen Kompetenzhochsignifikant sind.

- Was die Veränderungen in der Motivation und dem Selbstbild angeht, sind die Unterschiede zwischen den 
Veranstaltungen erheblich geringer und nur hinsichtlich der Leistungsmotivation auch signifikant. Deutliche Verbesserungen finden wir im Kurs 131, deutliche Verschlechterungen im Kurs 141.

Nimmt man alle Einzelergebnisse zusammen, dann unterscheiden sich die einzelnen Lehrveranstaltungen zum Teil erheblich hinsichtlich ihrer Vermittlung der Schlüsselkompetenzen sowie der Förderung der Leistungsmotivation, ansatzweise auch hinsichtlich der Verbesserung des Selbstbilds. Im Einzelnen fanden wir:

- überwiegend Verschlechterungen in den Veranstaltungen 141, 151, 321 und 211,

- überwiegend Verbesserungen in den Veranstaltungen 131 und 311 .

\subsection{Welchen Einfluss hat die Qualität der Lehre und des} Lernens auf die Veränderungen der Kompetenzen, der Motivation und der Selbstwahrnehmung in den einzelnen Veranstaltungen?

Wenn wir davon ausgehen, dass die Verschlechterungen und die Verbesserungen der Kompetenzen, der Motivation und der Selbstwahrnehmung ein Ergebnis der jeweiligen Qualität der Lehre und der Qualität des Lernens sind, dann sollten sich die Veranstaltungen systematisch darin unterscheiden.

Die Qualität der Lehre wird hier als umso höher betrachtet, je höher der Respekt, die Betreuung, Kompetenz, Motivierung und Didaktik sowie die Förderung der Gruppenarbeit der Dozenten. Die Variable Qualität der Lehre wird hier als Summenindex aus den o.g. Variablen gebildet und umfasst einen Wertebereich zwischen 25 und 72.

Die Qualität des Lernens wird hier als umso höher betrachtet, je intensiver die aktive Teilnahme, die Vor- und Nachbereitung der Lehrveranstaltungen der Studierenden und je intensiver der Transfer ihrer Inhalte erfolgt. Die Variable Qualität des Lernens wird ebenfalls als Summenindex aus den o.g. Variablen gebildet und umfasst einen Wertebereich zwischen 7 und 27.

Um herauszufinden, wie sich die Qualität des Lehrens und des Lernens in den Veranstaltungen unterscheiden, führen wir Varianzanalysen für beide Indizes durch, in denen die Veranstaltungsvariable als Faktor eingeht. Die Ergebnisse zeigen sich in den folgenden Mittelwertdiagrammen (vgl. Abb. 4 und 5) sowie im supplementary material, resource 2 Tab. A13.

Die deskriptive Analyse und die Varianzanalyse belegen, dass sich die Mittelwerte und Standardabweichungen der Qualität der Lehre der Lehrenden und der Qualität des Lernens der Studierenden zwischen den einzelnen Veranstaltungen erheblich und hochsignifikant unterscheiden. Die Fehlerbalken in den Abbs. 4 und 5, in denen auf der Basis der deskriptiven Analyse die Mittelwerte \pm 1 Standardfehler dargestellt sind, machen anschaulich deutlich, in welchen

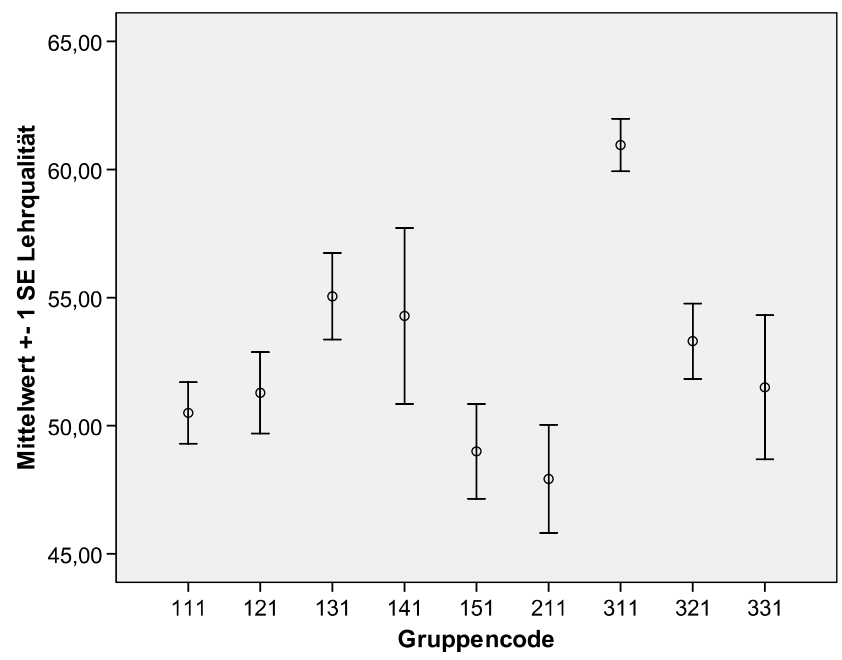

Abb. 4 Mittelwertvergleiche der Qualität der Lehre in den Veranstaltungen

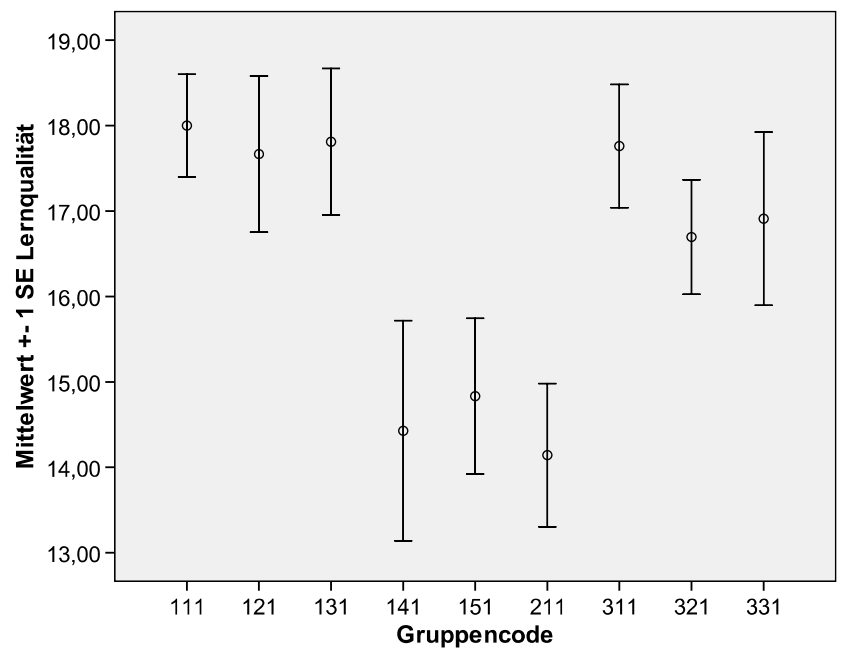

Abb. 5 Mittelwertvergleiche der Qualität des Lernens in den einzelnen Veranstaltungen

Veranstaltungen (Gruppencode) die Qualität der Lehre und des Lernens hoch bzw. niedrig sind.

Die Ergebnisse sind eindeutig:

- In den Veranstaltungen 131 und insbesondere 311 zeigen die Lehrenden nach der Einschätzung der Studierenden insgesamt die höchste Qualität in der Lehre. In genau diesen Kursen werden die Schlüsselkompetenzen, die Motivation und die Selbstwahrnehmung am stärksten verbessert.

- In diesen Veranstaltungen zeigen auch die Studierenden ein komplementäres Lernverhalten, indem sie aktiv mitarbeiten, die Veranstaltungen gut vor- und nachbereiten und sich auch außerhalb der Veranstaltungen über die Lehrinhalte unterhalten. Letzteres gilt auch noch für das Lernverhalten in den Veranstaltungen 111 und 121, in denen 
besonders die Interaktionskompetenzen verbessert werden.

- In den Veranstaltungen 151 und 211 finden wir eine relativ niedrig eingeschätzte Qualität der Lehre. Und genau in diesen Veranstaltungen finden eher Verschlechterungen als Verbesserungen bei den Schlüsselkompetenzen, speziell den Interaktionskompetenzen, sowie bei der Motivation und der Selbstwahrnehmung statt.

- Komplementär hierzu zeigen die Studierenden genau in diesen Veranstaltungen ein Lernverhalten, das nicht geeignet ist, ihre Kompetenzen, ihre Motivation und Selbstwahrnehmung zu verbessern. Die ebenfalls durchgehend beobachteten Verschlechterungen in der Veranstaltung 141 sind bei einer durchschnittlich eingeschätzten Lehrqualität überwiegend auf unzureichendes eigenes Lernverhalten der Studierenden zurückzuführen.

Insgesamt sind damit die Verbesserungen in den Kompetenzen, der Motivation und der Selbstwahrnehmung ein direktes Resultat der Qualität der Lehre der Lehrenden und der darauf bezogenen weitgehend komplementären Qualität des Lernens der Studierenden in den einzelnen Veranstaltungen: Je höher die Qualität der Lehre und je höher die damit verbundene Qualität des Lernens, desto stärker werden die Kompetenzen, die Motivation und die Selbstwahrnehmung verbessert.

4.5 Kann von der Zufriedenheit der Studierenden mit den Veranstaltungen bzw. den Veranstaltern auf die Qualität der Lehre geschlossen werden?

Die bisher beobachteten Unterschiede in der Vermittlung der Schlüsselkompetenzen, der Motivation und der Selbstwahrnehmung sowie der Lehrverhaltensweisen der Dozenten, aber auch der eigenen Lernverhaltensweisen sollten sich auch in unterschiedlichen Zufriedenheitsraten der Studierenden mit den Veranstaltungen und ihren Dozenten wider-

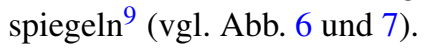

Wie nicht anders zu erwarten, entspricht das Bild der $\mathrm{Zu}$ friedenheit mit den Veranstaltungen im Großen und Ganzen dem Bild der Zufriedenheit mit den Veranstaltern.

Eine noch größere Übereinstimmung aber zeigt das Bild der Zufriedenheit mit den Lehrenden (Abb. 6) mit dem Bild, das sich aus den über Skalen gemessenen Lehrkompetenzen der Lehrenden (vgl. Abb. 4) ergibt: Beide Bilder sind nahe$z u$ identisch. Die über komplexe Skalen gemessenen Lehrkompetenzen eines Lehrenden korrelieren also nahezu perfekt mit der über eine einfache Frage erhobenen subjektiven Zufriedenheit der Studierenden mit ihm.

An dieser Stelle sei daher kurz auf die gerade von Lehrenden immer wieder geäußerte Skepsis in Bezug auf die

\footnotetext{
${ }^{9}$ Zufriedenheiten wurden für die Kontrollgruppe nicht erfragt.
}

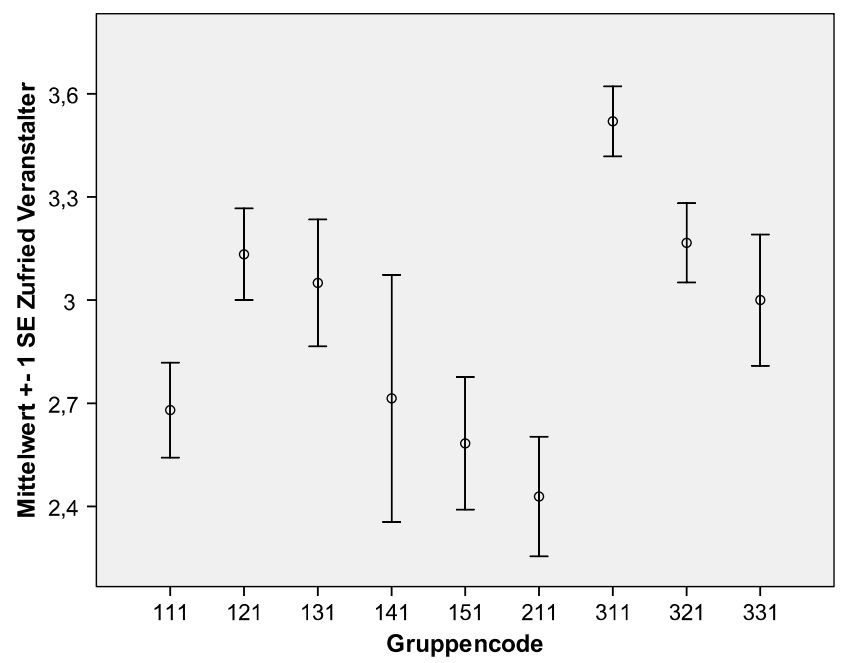

Abb. 6 Zufriedenheit der Studierenden mit dem Veranstalter

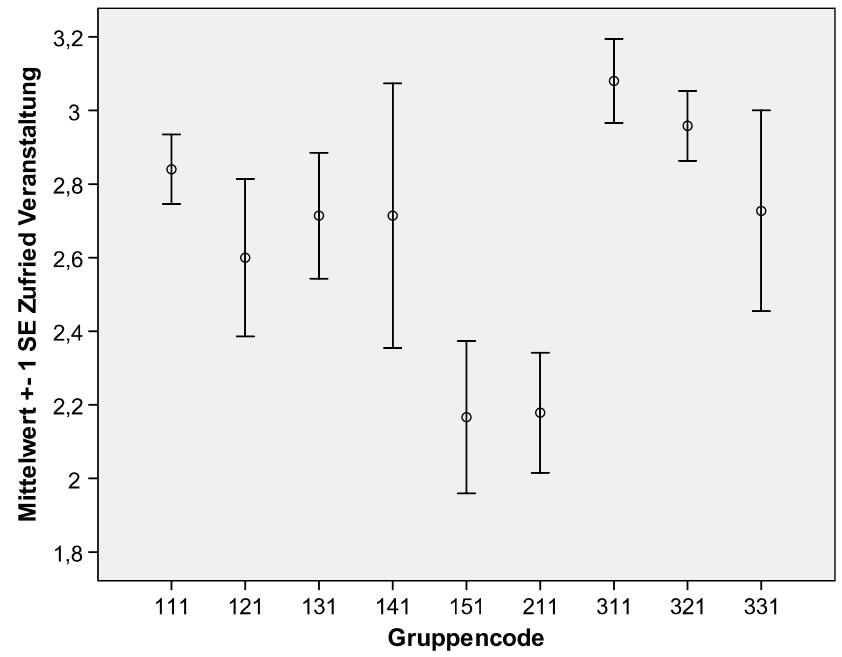

Abb. 7 Zufriedenheit der Studierenden mit der Veranstaltung

Gültigkeit studentischer Beurteilung eingegangen. Einschlägige Übersichtsstudien (Marsh 1982; Marsh und Roche 1997; Hennig 2006) kommen durchweg zu dem Ergebnis, „dass studentische Beurteilungen - über Veranstaltungen gemittelt - multidimensional, messgenau und stabil sind“" (Hennig 2006, S. 339f.). Studentische Beurteilungen stimmen in hohem Maße auch mit anderen Indikatoren für eine gute Lehre wie Fremdbeurteilungen und Leistungstests überein, und werden auch nicht von möglichen Verzerrungsvariablen, wie z.B. Geschlecht oder Intelligenz beeinflusst (Marsh und Roche 1997; Feldman 1989).

Es sollte jedoch auch darauf hingewiesen werden (Rindermann 2001), dass bei der Einschätzung des Lehrenden in der Regel auch Veranstaltungsaspekte mit gemessen werden. So werden, wie auch Esser (1994) zeigen konnte, z. B. Lehrende in Veranstaltungen mit institutionellbeschreibenden Inhalten deutlich besser bewertet als Leh- 
rende in Veranstaltungen mit abstrakt-theoretischen oder statistisch-methodischen Inhalten. Aus der Evaluation einer einzelnen Veranstaltung kann man daher auch nicht direkt auf die Lehrqualifikation eines Lehrenden schließen.

Insgesamt spiegeln die Ergebnisse der Fragen nach der Zufriedenheit die Ergebnisse der bisherigen Analysen wider: Sie passen genau in das Bild, das sich auch schon aus dem unterschiedlichen Lehr- und Lernverhalten und dessen Konsequenzen für die Veränderungen der Kompetenzen, der Motivation und der Selbstwahrnehmung gezeigt hatte.

\subsection{Zusammenfassung}

Zunächst einmal bleibt festzuhalten, dass sich die hochschulpolitischen Erwartungen, man könne mit eigens eingerichteten Kursen die hier angesprochen Kompetenzen der Studierenden zum selbstständigen Handeln und zur Interaktionskompetenz insgesamt direkt verbessern, nicht erfüllt haben. Auch hinsichtlich der Motivation sind keine systematischen Verbesserungen erkennbar. Allenfalls bezüglich der Selbstbilds lassen sich leichte Verbesserungen im Selbstwert und in der Selbstreflexion erkennen.

Zum zweiten ergeben sich deutliche Zusammenhänge zwischen der Verbesserung der Schlüsselkompetenzen, der Motivation und der Selbstwahrnehmung einerseits und der Qualität der Lehre sowie - in abgeschwächtem Maße - der Qualität des Lernens andererseits.

Dabei sind die Verbesserungen der Schlüsselkompetenzen vor allem auf die Verhaltensweisen der Lehrenden zurückzuführen, die ihre „Tüchtigkeit“ widerspiegeln: ihre fachliche Kompetenz, ihre didaktische Kompetenz und die Strukturiertheit der Veranstaltungen. Die Verbesserungen in der Motivation und im Selbstbild der Studierenden ist dagegen eher darauf zurückzuführen, wie respektvoll die Lehrenden mit ihren Studierenden umgehen, wie sie sie zu motivieren verstehen, in welchem Umfang sie sie betreuen und Kooperation und Gruppenarbeit fördern, insgesamt: ob sie in der Lage sind, ein positives sozial-emotionales Grundklima in der Veranstaltung herzustellen.

Die Verbesserungen in der Kompetenz zu selbstständigem Handeln, also zu aktiver Orientierung, zu zweckrationalem Handeln und zu selbstgesteuertem Lernen steigen vor allem mit einer qualitativ hochwertigen Lehre, in der die Lehrenden die Studierenden zu motivieren verstehen, in der sie ihnen mit Respekt entgegen treten und in der sie ihre Inhalte kompetent und strukturiert vermitteln und eine dem Lehrstoff adäquate Didaktik einsetzen, wobei sie die Studierenden dort abholen, wo sie stehen, und sich ihnen in der Geschwindigkeit ihres Lehrens anpassen; hinzu kommt ein umfassendes Betreuungsangebot.

Die beobachteten Verbesserungen in den Interaktionskompetenzen, z.B. der Kritikfähigkeit, der Kooperationsfähigkeit, den Fähigkeiten zum Perspektivenwechsel und zur
Empathie sind neben der Lehrqualität aber auch eine Folge des Studierendenverhaltens selbst, insbesondere des AusmaBes ihrer Einbindung in den Kreis der Kommilitonen innerhalb und außerhalb des Unterrichts. Je intensiver diese Einbindung ist und je produktiver und konstruktiver die Gruppe ist, desto stärker wachsen auch die Interaktionskompetenzen.

Wie weitergehende multivariate Analysen belegen (Lange 2009, S. 132ff.), kommt es in erster Linie auf das Lehrverhalten der Lehrenden an, dem das Lernverhalten der Studierenden weitgehend folgt, indem sie sich aktiv in die Veranstaltungen einbringen, die Veranstaltungen gründlich vor- und nachbereiten und sich auch außerhalb der Veranstaltungen inhaltlich mit dem Stoff auseinandersetzen. Dieses Ergebnis bestätigt frühere Ergebnisse der Unterrichtsforschung allgemein (Lange 2005) sowie der Hochschulforschung im Besonderen (Rindermann 2001).

Zum dritten zeigt die veranstaltungsspezifische Analyse, dass sich die Veranstaltungen hinsichtlich der Veränderungen der Schlüsselkompetenzen, insbesondere der Kompetenz zu selbstständigem Handeln und der allozentrischen Interaktionskompetenz der Studierenden erheblich und hochsignifikant unterscheiden. Die Unterschiede sind geringer hinsichtlich der Veränderung der Motivation und der Selbstwahrnehmung. Wir finden insgesamt also Veranstaltungen mit deutlichen Verbesserungen auf nahezu allen Dimensionen, andere mit deutlichen Verschlechterungen und wiederum einige wenige Veranstaltungen, in denen sich gar nichts verändert hat. Damit neutralisieren sich Verbesserungen und Verschlechterungen über alle Veranstaltungen gesehen.

Vergleicht man zum vierten die Veranstaltungen mit Verbesserungen bzw. Verschlechterungen in den Schlüsselkompetenzen, der Motivation und der Selbstwahrnehmung mit der dort berichteten Lehr- bzw. Lernqualität, dann bleibt festzuhalten: Veranstaltungen, in denen wir Verbesserungen in den Schlüsselkompetenzen feststellen, sind zugleich diejenigen, die sich durch eine hohe Qualität der Lehre sowie, daran anschließend, durch eine hohe Qualität des Lernens auszeichnen. Umgekehrt finden wir in den Veranstaltungen, in denen wir nur geringe Verbesserungen oder gar Verschlechterungen in den Schlüsselkompetenzen finden, eine relativ geringe Einschätzung der Qualität der Lehre und der Qualität des Lernens.

Ein bemerkenswertes Nebenresultat bleibt zu erwähnen: Die ,einfachen“ Einschätzungen der Zufriedenheit der Studierenden mit den Veranstaltern bilden deren gemessene Lehrkompetenzen bereits sehr gut ab.

\section{Hochschuldidaktische Konsequenzen}

Wer hochschuldidaktische Konsequenzen aus den Ergebnissen dieser Studie formulieren will, muss sich an den 
Zielsetzungen oder allgemeiner an der Programmatik der Hochschulausbildung orientieren. Darüber aber herrscht gegenwärtig offener Streit, der sich in zwei einander gegenüberstehenden, hier apodiktisch wiedergegebenen Positionen manifestiert.

Auf der einen Seite stehen die Befürworter der klassischen Universität, die eine wissenschaftliche Ausbildung der Studierenden in den Vordergrund stellen, in der die Studierenden lernen, in der Gemeinschaft der Lehrenden und Lernenden selbstständig wissenschaftliche Erkenntnisse nach den Regeln z.B. des Kritischen Rationalismus oder auch der Kritischen Theorie zu erarbeiten. Gefördert werden sollen hier vor allem die selbstständige systematische Erkenntnisproduktion und die kritische Auseinandersetzung mit den erzeugten Erkenntnissen. Das verlangt ein hohes $\mathrm{Ma} ß$ an Selbstständigkeit und Offenheit aller Beteiligten, an kritischem Diskurs und Kooperation zwischen Lehrenden und Lernenden zwischen im Prinzip Gleichen. Abgelehnt werden in dieser Position sowohl eine direkte staatliche Einflussnahme auf die Inhalte und Vorgehensweisen des Erkenntnisprozesses als auch eine Einflussnahme der Wirtschaft und Verwaltung, die die Vermittlung der Inhalte und ihrer Produktion wirtschaftlichen Verwertungsinteressen unterstellen und am Ausbildungsziel der ,employability" ausrichten möchten. Diese Position steht in der Tradition der klassischen Universität, wie sie z.B. von Humboldt oder von Schleiermacher vertreten, und z.B. in einem neueren Beitrag von F. Grigat (2008) in „Forschung und Lehre“, der Zeitschrift des Hochschulverbandes, formuliert wurde.

Auf der anderen Seite stehen die Befürworter der Universität als einer Ausbildungsinstitution, in der die Studierenden wie an einer Fachhochschule vor allem berufsbezogenes, praktisch verwertbares Wissen erwerben sollen, ohne am Prozess der Wissensgenerierung selbst aktiv beteiligt zu sein und ihn kritisch zu hinterfragen. Die Inhalte werden daher auch an Anforderungen aus möglichen zukünftigen Berufsfeldern orientiert. Der Ausbildungsprozess wird bürokratisch-hierarchisch organisiert, die Lehrenden werden entweder als Vorgesetzte der ihnen untergebenen Studierenden oder aber als Verkäufer der Ware Wissen begriffen, denen die Studierenden als Kunden gegenüber stehen, die für den Erwerb ihres demnächst beruflich verwertbaren Wissens auch Studiengebühren zu zahlen haben. Staat und Wirtschaft bestimmen in den Universitätsräten als den höchsten Entscheidungsgremien über die inhaltlichen Lehrund Forschungsprogramme, die Organisation der Hochschule, das Personal und über die methodisch-didaktische Vorgehensweise entscheidend mit. Diese Position findet sich im Bologna-Abkommen und den Ausführungen der Bolognanachfolgekonferenzen, sie wird von der Hochschulrektorenkonferenz unterstützt und findet ihren Niederschlag in den gegenwärtigen Hochschulgesetzen der Bundesrepublik.

Welche Position man auch immer vertritt - der Autor neigt hier eindeutig zur ersten Position -, Einigkeit dürfte unter allen mit der universitären Ausbildung Befassten darin bestehen, dass im pädagogischen Prozess sowohl fachliche, und damit auch berufsqualifizierende Inhalte vermittelt und erworben werden sollen, als auch überfachliche Kompetenzen, wie z.B. die Schlüsselkompetenzen zur Persönlichkeitsbildung im weiteren Sinne erzeugt werden sollen. Die Frage ist dabei allerdings, in welcher relativen Gewichtung und wie organisiert: als formeller Ausbildungs- oder als informeller Lern- und Erfahrungsprozess?

Die Art der vertretenen Position hat selbstverständlich Auswirkungen auch auf die hochschuldidaktischen Konsequenzen, die aus dieser Studie zu ziehen sind. Diese Auswirkungen müssen auch den Staats- und Regierungschefs bereits bewusst gewesen sein, als sie mit dem BolognaAbkommen explizit die Einführung von Kursen zur Vermittlung von Schlüsselkompetenzen einforderten. Offensichtlich haben sie bereits geahnt, dass mit der Modularisierung und Verschulung der Universitätsausbildung Gefahren für die Kompetenz zu selbstständigem Handeln und für die Interaktionskompetenz der Studierenden sowie für deren kritische Auseinandersetzung mit dem Prozess der Erkenntnisgenerierung und Erkenntnisvermittlung ausgehen könnten.

$\mathrm{Da}$ an dieser Stelle weder argumentativ auf die o.g. Positionen noch systematisch auf alle möglichen Aspekte der Hochschuldidaktik eingegangen werden kann, die z.B. Lehrbücher ansprechen (vgl. z.B. Macke et al. 2008), möchte ich nur einige Konsequenzen aus dieser Studie benennen, über die man dann diskutieren kann. Dabei ist zu bedenken, dass hier auf die Schlüsselkompetenzen abgestellt wird, die von der OECD für wichtig gehalten wurden, von der sich die Definitionen vieler deutscher Hochschulen allerdings erheblich unterscheiden.

- Bei aller Unterschiedlichkeit in der Definition der Schlüsselkompetenzen besteht doch Konsens darin, dass Studierende in die Methoden und Techniken wissenschaftlichen Arbeitens und der dafür zur Verfügung stehenden Instrumente eingeführt werden sollen, dazu gehören auch der Umgang mit dem Computer und seinen Softwareprogrammen, die Nutzung von Bibliotheken und von Elearning-Plattformen. All diese Kompetenzen lassen sich sicherlich in Veranstaltungen vermitteln und erwerben, die unabhängig von den Fakultäten auf der Ebene der Universität durchgeführt werden können.

- Darüber hinaus besteht sicherlich auch noch Konsens darüber, dass für die einzelnen Fachgebiete wissenschaftspropädeutische Kompetenzen vermittelt und erworben werden sollen. Hierzu gehören z.B. in den naturwissenschaftlichen Fächern die Kenntnis und die kritische Auseinandersetzung mit experimentellen Anordnungen, in den Wirtschafts- und Sozialwissenschaften die Einführung in Modellbildung und Simulationen, in Verfahren der Datenerhebung und Datenanalyse sowie in statistische Verfahren, in den Geisteswissenschaften 
die Kenntnis hermeneutischer Verfahren. All diese wissenschaftspropädeutischen Verfahren lassen sich in fachspezifischen oder in fachgruppenspezifischen Veranstaltungen vermitteln und erwerben. Auch Fremdsprachenkompetenzen, die über die grundlegenden Kompetenzen hinaus fachspezifisch oder fachgruppenspezifisch anzulegen sind, können über derartige Veranstaltungen vermittelt werden.

- Ganz anders sieht es dagegen mit der hier näher untersuchten Kompetenz zum selbstständigen Handeln und der Interaktionskompetenz, aber auch mit der Motivation der Studierenden und ihrer Selbstwahrnehmung aus. Gleichgültig, ob man sie explizit zum Thema einer Veranstaltung macht oder sie implizit vermittelt, sie können theoretisch und praktisch in jeder Form einer Lehrveranstaltung, seien es Veranstaltungen zu allgemeinen Methoden und Techniken, zur Propädeutik oder zu fachspezifischen Inhalten vermittelt und erworben werden, sofern die Qualität der Lehre stimmt, dem das entsprechende Lernverhalten der Studierenden folgt. Damit sind wir bei den Lehrenden und ihrer didaktisch-methodischen Kompetenz.

- Die Studie hat zum einen das Ergebnis erbracht, dass, über alle untersuchten Veranstaltungen gesehen, keine systematischen Verbesserungen in den hier untersuchten Schlüsselkompetenzen erreicht wurden. Damit wird man daran zweifeln müssen, ob der hier gewählte Ansatz, bei dem die Lehrenden durch Multiplikatoren für die Vermittlung der Schlüsselkompetenzen geschult werden, für die Vermittlung der hier infrage stehenden Schlüsselkompetenzen brauchbar ist; möglicherweise muss hier aber auch nur die Schulung verbessert werden. Die Studie hat aber zum anderen das Ergebnis erbracht, dass sich die einzelnen Veranstaltungen erheblich hinsichtlich der Veränderungen der Schlüsselkompetenzen unterscheiden, und dass die Verbesserung oder Verschlechterung vor allem ein Ergebnis der Qualität der Lehre sind, dem die Qualität des Lernens folgt.

- Daraus folgt, dass in erster Linie die Lehrkompetenz der Lehrenden gefördert werden muss, wenn man die Schlüsselkompetenzen der Studierenden verbessern will. Je besser nämlich die Lehre, desto erfolgreicher ist auch das studentische Lernen und desto eher werden dann ihre Schlüsselkompetenzen sowie generell auch ihre fachlichen Kompetenzen gefördert. Wodurch sich „gute“ Universitätslehre auszeichnet, wird in jedem guten Lehrbuch zur Hochschuldidaktik dargestellt (z.B. Macke et al. 2008). Weitere Hinweise ergeben sich aus dieser Studie direkt überall dort, wo deutliche und signifikante Zusammenhänge zwischen der Verbesserung der Schlüsselkompetenzen und dem Lehrverhalten der Lehrenden aufgezeigt wurden. Umstritten ist jedoch, wie die Hochschullehrer und wissenschaftlichen Mitarbeiter ihre Methodik und Didaktik lernen (sollten), wenn sie hierzu nicht ver- pflichtet sind, sondern sich, wie heutzutage noch üblich, als geborene Lehrer verstehen.

- Eine Möglichkeit für Hochschullehrer und wissenschaftliche Mitarbeiter ist gegenwärtig das Selbststudium anhand von Lehrbüchern zur Hochschuldidaktik. Der Ertrag wird allerdings suboptimal sein, da keine Rückmeldung durch Dritte erfolgt.

- Eine zweite Möglichkeit existiert für wissenschaftliche Mitarbeiter, aber auch für Professoren, im Teamteaching von den Erfahrungen der Älteren bzw. der Kolleginnen und Kollegen zu lernen. Diese Möglichkeit wird aber aktuell durch die Kapazitätsverordnungen behindert, nach denen etwa eine zweistündige Lehrveranstaltung bei zwei Lehrenden nur jeweils eine Stunde in Anrechnung bringen lässt, eine äußerst prohibitive Regelung für das Teamteaching.

- Eine dritte Möglichkeit ist die Teilnahme der Lehrenden an Veranstaltungen der hochschuldidaktischen Zentren, die in vielen Universitäten derzeit eine Renaissance erfahren (Battaglia 2008). Die Erfahrung zeigt, dass bereits der Besuch einiger weniger guter Didaktikveranstaltungen die Lehrkompetenz der Lehrenden erheblich zu steigern vermag; hierzu tragen auch Supervisionen der eigenen Veranstaltungen durch einen Didaktiker bei. Die Inanspruchnahme derartiger Angebote wird man für die „Anfänger“ in der Lehre sicherlich mit einem höheren Verbindlichkeitsgrad ausstatten können als für die „Erfahrenen“, für die es systematische Anreize zu setzen gilt.

Bleibt an dieser Stelle, auf einige mit der Einführung der modularisierten Ausbildungsgänge auftretende Risiken für die Vermittlung der Schlüsselkompetenzen, der intrinsischen Lernmotivation und der Leistungsmotivation hinzuweisen. Mit der Modularisierung und der Verschulung sowie der Straffung der Studiengänge werden den Studierenden Freiräume für die Entwicklung der Kompetenz zum selbstständigen Handeln genommen. Erste Erfahrungsberichte zur Einführung der BA-Studiengänge zeigen auch, dass Zweifel an der von diesen „Reformen“ erwarteten Verkürzung der Studiendauer, der Senkung der Abbrecherzahlen (Heublein et al. 2008) sowie der Steigerung der Mobilität (BolognaZentrum der HRK 2008) bestehen.

Mit der Einführung der credit-points und der Ausrichtung der Studiengänge am Ziel der employability wird die intrinsische Lernmotivation behindert und der Aufbau extrinsischer Orientierungen verstärkt. Generell wird die Ausbildung damit verstärkt unter das Primat sowohl der Bürokratie als auch der Wirtschaft gestellt. Beide Ausrichtungen behindern tendenziell den Aufbau einer intrinsischen Lernorientierung und den Aufbau von Leistungsmotivation. Dabei wären auch für die Wirtschaft Arbeitskräfte mit einer starken intrinsischen Orientierung und einer hohen Leistungsmotivation langfristig gewinnbringender als Arbeitskräfte, die sich nur an äußeren (materiellen) Kriterien orientieren. 
Aber auch für die Universitäten entsteht die Gefahr, dass sich das zukünftige Hochschulpersonal stärker vom Prozess der intrinsisch orientierten Erkenntnisgewinnung abwendet und nur noch danach fragt, in welchen Forschungs- und Lehrfeldern kurzfristig materieller Erfolg und/oder Statusgewinn erzielt werden können.

Unter dem Aspekt der Evaluation von Lehrveranstaltungen bleibt darauf hinzuweisen, dass sich das methodische Inventar zur Erhebung der Schlüsselkompetenzen und ihrer Unterrichtsbedingungen offensichtlich bewährt hat und zukünftig eingesetzt werden kann, um zu überprüfen, inwieweit in einzelnen Lehrveranstaltungen Schlüsselkompetenzen sowie Motivation und Selbstwahrnehmung vermittelt werden.

Ein wichtiges Forschungsdesiderat bleibt allerdings bestehen: $\mathrm{Ob}$ und unter welchen Bedingungen die hier mit Hilfe von Selbsteinschätzungsskalen erhobenen Schlüsselkompetenzen als individuelle Ressourcen auch faktisch zu selbstständigem Handeln und zur gewünschten Interaktion und Kooperation führen, bleibt offen. Hier müssten Untersuchungen durchgeführt werden, in denen das Handeln der Studierenden in konkreten Situationen beobachtet wird, um die prognostische Qualität der Kompetenzskalen einschätzen zu können. Vor diesem Hintergrund müssen auch die von den Studierenden selbst berichteten Kompetenzen relativiert werden.

Danksagung Für wertvolle Hinweise zur Überarbeitung des Manuskripts danke ich der Redaktion und den anonymen Gutachtern.

\section{Literatur}

Battaglia, S.: Auf dem Vormarsch. Die Hochschuldidaktik bahnt sich ihren Weg. Forsch. Lehre 9, 602-603 (2008)

Bologna-Zentrum der HRK: Mobilität im Studium. Eine Untersuchung zu Mobilität und Mobilitätshindernissen in gestuften Studiengängen innerhalb Deutschlands. Bonn (2008)

Buschor, C.B., Forrer, E.: Überfachliche Kompetenzen junger Erwachsener am Übergang zwischen Schule und Beruf: Jugend- und Rekrutenbefragung als Beitrag zum Bildungsmonitoring. Dissertation, Universität Zürich (2005)

Coleman, J.S.: Grundlagen der Sozialtheorie, Handlungen und Handlungssysteme, Bd. 1. Oldenbourg, München (1995)

Deci, E.L.: Intrinsic Motivation. Plenum, New York (1975)

Deci, E.L., Ryan, R.M.: The "what" and "why" of goal pursuits: human needs and the self-determination of behavior. Psychol. Inq. 11, 227-268 (2000)

Esser, H.: Lehrbericht der Fakultät für Sozialwissenschaften der Universität Mannheim. Ergebnisse der Studenten- und Lehrerhebung im Wintersemester 1993/94. Mannheim, Universität Mannheim, Fakultät für Sozialwissenschaften (1994)

Esser, H.: Soziologie. Spezielle Grundlagen, Bd. 1. Campus, Frankfurt (1999)

Feldman, K.A.: Instructional effectiveness of college teachers as judged by teachers themselves, current and former students, col- leagues, administrators and external (neutral) observers. Res. High. Educ. 30, 137-194 (1989)

Fend, H.: Theorie der Schule. Urban \& Schwarzenberg, München (1980)

Fittkau, B.: Beobachtung und Beurteilung von Hochschulunterricht. In: Berendt et al., Hochschuldidaktik: Lehren und Lernen im Hochschulalltag. Otto Müller Verlag, Salzburg (1979)

Grigat, F.: „Erkennen nicht lernen ist der Zweck der Universität“. Fragen zur Qualität der Lehre an Friedrich Schleiermacher. Forsch. Lehre 9, 592-594 (2008)

Grob, U., Maag Merki, K.: Überfachliche Kompetenzen. Theoretische Grundlegung und empirische Erprobung eines Indikatorensystems. Peter Lang, Bern (2001)

Grob, U., Maag Merki, K.: Überfachliche Kompetenzen: Zur Validierung eines Indikatorensystems. Empir. Pädagog. 17, 123-147 (2003)

Heckhausen, H.: Hoffnung und Furcht in der Leistungsmotivation. Hain, Meisenheim (1963)

Heckhausen, H.: Motiv und Motivation. In: Hermann, T., et al. (Hrsg.) Handbuch psychologischer Grundbegriffe, S. 296-313. KöselVerlag, München (1977)

Heckhausen, H.: Motivation und Handeln. Springer, Berlin (1989)

Hennig, M.: Evaluation der Lehre - theoretische und methodische Aspekte. Soziologie 35, 333-348 (2006)

Heublein, U., Schmelzer, R., Sommer, D., Wank, J.: Die Entwicklung der Schwund- und Studienabbruchquoten an den deutschen Hochschulen. Projektbericht Mai, HIS, Hannover (2008)

Lange, E.: Theoretisches Modell zur Beschreibung und Erklärung von „erfolgreichem“ Hochschulunterricht. Bielefeld, Ms. (1992)

Lange, E.: Jugendkonsum im 21. Jahrhundert. Eine Untersuchung der Einkommens-, Konsum- und Verschuldungsmuster der Jugendlichen in Deutschland. VS Verlag für Sozialwissenschaften, Wiesbaden (2004)

Lange, E.: Soziologie des Erziehungswesens. VS Verlag für Sozialwissenschaften, Wiesbaden (2005)

Lange, E.: Schlüsselkompetenzen - Wie sie entstehen und verbessert werden können. Eine empirische Untersuchung bei Studierenden. Verlag Barbara Budrich, Opladen (2009)

Macke, G., Hanke, U., Viehmann, P.: Hochschuldidaktik: lehren, vortragen, prüfen. Weinheim, Beltz (2008)

Marsh, H.W.: Validity of students' evaluations of college teaching: a multitrait-multimethod analysis. J. Educ. Psychol. 74, 264-279 (1982)

Marsh, H.W., Roche, L.A.: Making students' evaluations of teaching effectiveness effective: the central issue of validity, bias, and utility. Am. Psychol. 52, 1187-1197 (1997)

Mummendey, H.D.: Psychologie des „Selbst“. Hogrefe, Göttingen (2006)

Rychen, D.S., Salganik, L.H. (Hrsg.): Defining and Selecting Key Competencies. Hogrefe \& Huber, Göttingen (2001)

Rychen, D.S., Salganik, L.H. (Hrsg.): Key Competencies for a Successful Life and a Well-Functioning Society. Hogrefe \& Huber, Göttingen (2003)

Rindermann, H.: Die studentische Beurteilung von Lehrveranstaltungen - Forschungsstand und Implikationen. In: Spiel, C. (Hrsg.) Evaluation universitärer Lehre - zwischen Qualitätsmanagement und Selbstzweck, Waxmann, Münster, S. 61-88 (2001)

Schiefele, U., Köller, O.: Intrinsische und extrinsische Motivation. In: Rost, D.H. (Hrsg.) Handwörterbuch Pädagogische Psychologie, S. 303-310. Beltz, Weinheim (2006) 


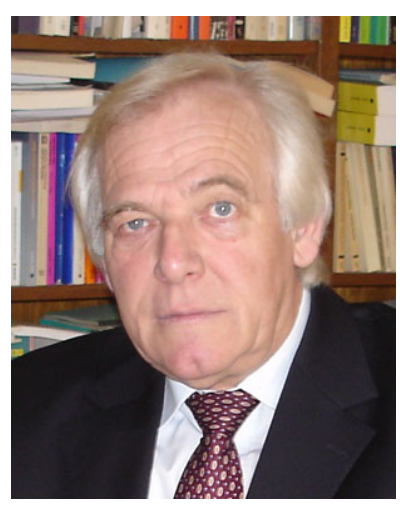

Elmar Lange geboren 1943, Universitätsprofessor für Soziologie an der Fakultät für Soziologie der Universität Bielefeld, i.R. Lehr- und Forschungsschwerpunkte: Sozialstrukturanalyse, Wirtschaftssoziologie, Bildungssoziologie,Jugendkonsum und Methoden empirischer Sozialforschung Letzte Veröffentlichungen:

(1) Elmar Lange (2009), Schlüsselkompetenzen - Wie sie entstehen und verbessert werden können, Opladen: Verlag Barbara Budrich.

(2) Karin R. Fries/Peter H. Göbel/Elmar Lange (2007), Teure Jugend. Wie Teenager kompetent mit Geld umgehen, Opladen: Verlag Barbara Budrich.

(3) Elmar Lange (2005), Soziologie des Erziehungswesens, Wiesbaden 2005: VS Verlag für Sozialwissenschaften. 